\title{
Totally Organic Polymer-Based Electrochromic Cell Using TEMPO-Substituted Polynorbornene as a Counter Electrode-Active Material
}

\author{
By Yusuke TAKAHASHI, ${ }^{1}$ Naoki HAYASHI, ${ }^{1}$ Kenichi OYAIZU, ${ }^{1}$ Kenji HONDA, ${ }^{2}$ and Hiroyuki NISHIDE ${ }^{1, *}$
}

\begin{abstract}
An electrochromic (EC) cell using a viologen-based polymer as an EC material and a radical polymer bearing a redox-active 2,2,6,6-tetramethylpiperidin- $N$-oxyl (TEMPO) group per repeating unit as a counter electroactive material was fabricated. The radical polymer was spin-coated on an ITO/glass electrode as the counter electrode of the EC cell. The electrochromic material of the cell was a polyion complex consisted of poly(decyl viologen) and poly(styrene sulfonate) (PV10-PSS), which was also spin-coated on the ITO/glass. An ion-conducting polymer gel solution was sandwiched between the two electrodes. Electrochemical switching of the cell was monitored using the visible absorption of the PV10-PSS complex $\left(\lambda_{\max }=550 \mathrm{~nm}\right)$ that appeared in the reduced state, while the radical polymer was transparent in the visible region in both redox states. PV10PSS and the radical polymer were concurrently reduced and oxidized, respectively, on each electrode during the charging process, which corresponded to the coloration of the cell. The decoloration of the polyion complex was effected by a discharging process under short circuit conditions. The electrochromic behavior of the cell was characterized by a remarkably low driving voltage, as a result of a small potential gap between PV10-PSS and the radical polymer. The use of the organic redox polymers, not only for the low energy-driven electrochromic switching but also for the charge-storage purposes, allowed a universal design of a battery-like display device, with possible application to a flexible and totally organic electrochromic cell.

KEY WORDS: Electrochromism / Organic Redox Polymer / Radical Polymer / Battery /
\end{abstract}

The electrochromism of organic and inorganic molecules have attracted much attention, with a view to apply them to windows and glasses. ${ }^{1}$ Electrochromic (EC) cells exhibit color changes, by applying specific driving voltages to cause the redox reaction of the EC materials attached to the surface of the transparent electrodes. However, there are some problems that must be overcome before consideration of practical use, such as the improvement of switching speed and cycle stability. The previously reported EC cells for display applications have mostly relied on using inorganic EC materials, such as prussian blue and $\mathrm{WO}_{3}{ }^{1,2} \mathrm{~A}$ number of efforts to improve the stability and the life of the EC cells have been made, by incorporating redox-active materials to the counter electrode, expecting a battery-like charge-storage configuration. ${ }^{3}$ Typical examples include the use of $\mathrm{Fe}_{2}\left(\mathrm{WO}_{4}\right)_{3}$-containing graphite as the counter electrode for the $\mathrm{WO}_{3}$-based $\mathrm{EC}$ cells, ${ }^{4}$ and the incorporation of anti-polarization agents such as metal oxides (i.e., $\mathrm{MnO}_{x}, \mathrm{VO}_{x}$, and $\mathrm{WO}_{x}$ ) to prevent side reactions (e.g., hydrogen gas generation) on cathodes.

Organic polymers have also been studied as EC materials which are almost free from the side reactions, by virtue of the relatively low driving voltages., ${ }^{2,5}$ For example, viologenbased polymers such as poly(alkyl viologen)s have been reported for their EC properties, due to their brilliant color changes between violet and transparent states. ${ }^{7}$ The viologen polymers are dialkylene salts of bipyridinium units, and they are usually insoluble in organic solvents but soluble in water. For EC applications, the viologen polymers are coated on an electrode as a multilayer or in the form of a polymer complex with a polyanion such as poly(styrene sulfonate) (PSS). ${ }^{8-10}$ The use of polyion complexes ${ }^{11}$ has successfully led to the fabrication of a polymer film which persist in water without loosing electrochromic properties, such as a series of poly(sodium styrenesulfonate)-poly(aklylene viologen) complexes, ${ }^{9}$ and those prepared from pendant-type viologen polymers. $^{10}$

We anticipated that the property of the viologen-based EC cell would be much improved by applying a battery-like configuration using a redox-active but electrochromically inert polymer layer at the surface of the counter electrode. The use of the counter electrode-active material originates from our recent findings that a combination of organic radical polymers with a conventional inorganic EC material, prussian blue (PB), allowed a novel design of an EC cell with a battery-like charge/discharge characteristics. ${ }^{12}$ Here, the radical polymer is an organic linear polymer bearing robust radical substituents as a pendant group per repeating unit of aliphatic backbones, which are characterized by rapid and reversible charge storage, ${ }^{13}$ transparent film formability and capability of controlling the redox potential based on the substituent effect. ${ }^{14}$ We have reported the application of the radical polymers to a rechargeable battery as electroactive materials on electrodes,

\footnotetext{
${ }^{1}$ Department of Applied Chemistry, Waseda University, Tokyo 169-8555, Japan

${ }^{2}$ Advanced Research Center for Science and Engineering, Waseda University, Tokyo 169-8555, Japan

*To whom correspondence should be addressed (Tel: +81-3-3200-2669, Fax: +81-3-3209-5522, E-mail: nishide@ waseda.jp).
} 


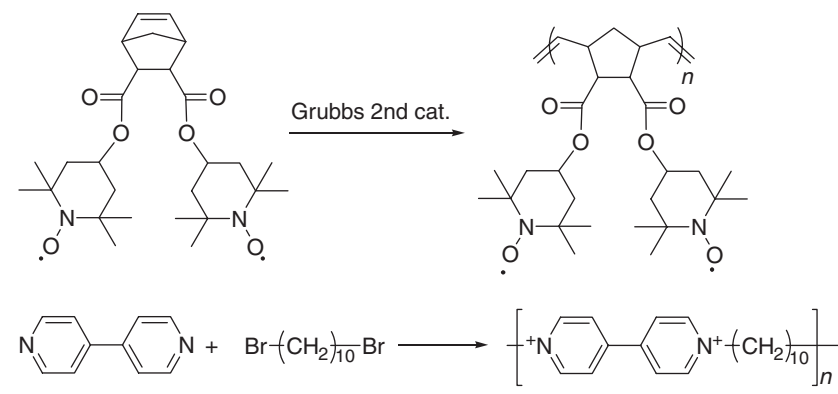

Scheme 1.

taking advantage of their redox-isolated electrochemical properties. ${ }^{15-18}$ In this paper, we report the results of our continuous attempts to develop totally organic EC cell, by exploring an organic EC material that is matched to couple with the radical polymers in terms of color and driving voltages.

\section{EXPERIMENTAL}

\section{Preparation of the Radical Polymer}

The norbornene monomer bearing the 2,2,6,6-tetramethylpiperidinoxyl (TEMPO) radical substituent, shown in Scheme 1, was synthesized from 5-norbornene-2,3-dicarboxylic anhydride by the previously reported procedure ${ }^{19}$ Conventional ring-opening metathesis polymerization of the monomer with the Grubbs second generation catalyst gave the corresponding radical polymer, poly[2,3-bis(2,2,6,6-tetramethylpiperidine- $N$-oxycarbonyl)-norbornene], in a high yield $(80 \%)$ $\left(M_{\mathrm{n}}=1.1 \times 10^{4}, M_{\mathrm{w}} / M_{\mathrm{n}}=1.1\right)$. The polymer was soluble in THF, toluene and dichloromethane, but insoluble in methanol and diethyl ether. An ethyl lactate solution of the polymer was spin-coated on an ITO/glass substrate.

\section{Synthesis of Poly(decyl viologen) and Complexation with a Counterion Polymer}

The EC polymer, poly(decyl viologen) (PV10), was synthesized by the previously reported procedure (Scheme 1). ${ }^{8} 4,4^{\prime}$ Bipyridine and 1,10-dibromodecane was reacted in a methanol/DMF mixture at $60^{\circ} \mathrm{C}$ for overnight. The resulting yellow precipitation was collected, washed with chloroform and dried under vacuum to yield PV10 as a yellowish powder in $73 \%$ yield. The degree of polymerization was determined by the ${ }^{1} \mathrm{H}$ NMR spectrum to be $c a$. 10 , using the peaks ascribed to the protons on the terminal pyridyl group at 8.8, 8.7, 8.3 and $7.8 \mathrm{ppm}$, and those of the pyridyl groups in the main chain around 9.0 and $8.4 \mathrm{ppm}$.

The complex of PV10 with poly(styrene sulfonate) (PSS) was prepared by adding a $5 \mathrm{wt} \%$ aqueous solution of PSS $\left(M_{\mathrm{w}}=75000,18 \%\right.$ solution obtained from Aldrich) into a $5 \mathrm{wt} \%$ aqueous solution of PV10 in a molar ratio of 2:1. The obtained precipitate was collected by filtration and dried under vacuum to give a light yellow powder in $50 \%$ yield. The polymer complex was dissolved in a mixture of $\mathrm{HCl}$, water and

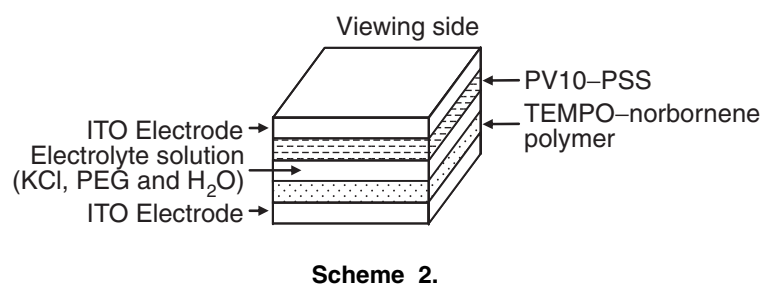

dioxane in a weight ratio of 5:50:45, and spin-coated onto an ITO/glass substrate.

\section{Fabrication of the EC Cell}

An electrolyte solution was placed between the two electrodes, which consisted of poly(ethylene glycol) (PEG, $M_{\mathrm{w}}=5.0 \times 10^{5}$, obtained from Wako), $\mathrm{KCl}$ and water with a weight ratio of 5:10:100. An EC cell was set up by stacking the radical polymer film, the electrolyte solution using a silicone rubber separator with a thickness of $2 \mathrm{~mm}$, and the PV10-PSS complex film as the EC layer, as shown in Scheme 2. The EC cell was characterized by using a PARSTAT 2263 Advanced Electrochemical System (Princeton Applied Research) for cyclic voltammometry (CV) and V-550 UV/vis spectrophotometer (Jasco) for color changes associated with the redox of PV10-PSS.

\section{RESULTS AND DISCUSSION}

Cyclic voltammogram of PV10-PSS in an aqueous electrolyte solution of $\mathrm{KCl}$ showed a reversible wave at $-0.17 \mathrm{~V}$, due to the $1 \mathrm{e}^{-}$reduction of the viologen unit (Figure 1 ). The amount of charges consumed during the potential scan was $7 \mathrm{mC}$ based on the integration of the redox wave, which corresponded to the redox capacity of the PV10-PSS layer. The theoretical capacity, based on the formula-weight based calculated amount using the thickness of the layer $(80 \mathrm{~nm})$, was $6 \mathrm{mC}$ which was in good agreement with the experimental value, suggesting that almost all of the viologen unit acted as the redox active site. The coincidence of the experimental and calculated values for the redox capacity strongly supported that (a)
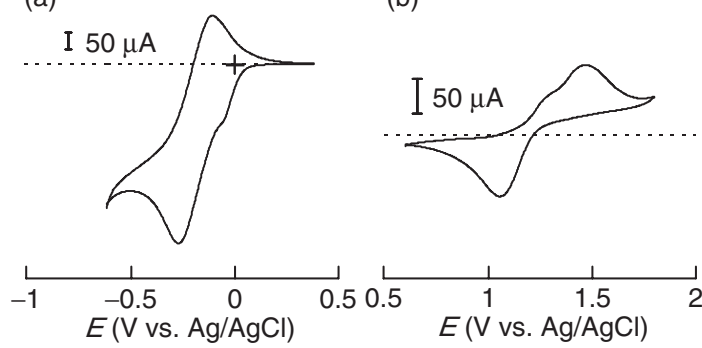

Figure 1. Cyclic voltammograms of the PV10-PSS layer $(80 \mathrm{~nm}$ thick) (a) and the TEMPO-radical polymer layer $(210 \mathrm{~nm}$ thick) (b) on the ITO/glass electrode immersed in aqueous solution of $\mathrm{KCl}(0.1 \mathrm{M})$. The effective electrode area was $5 \mathrm{~cm}^{2}$ for both electrodes. The counter electrode was a Pt wire, and the reference electrode was $\mathrm{Ag} / \mathrm{AgCl}$. Sweep rate $=10 \mathrm{mV} / \mathrm{s}$. 
the redox wave in Figure 1(a) was due to the $1 \mathrm{e}^{-}$reduction of the viologen unit to the corresponding cation radical, rather than the $2 \mathrm{e}^{-}$reduction to the quinoid state that proceeds at potentials more negative than $-0.5 \mathrm{~V}$, according to the previous report. ${ }^{8}$ The diffusion tail-like behavior in Figure 1(a) is the result of both residual $\mathrm{O}_{2}$ in the electrolyte solution that undergoes reduction near $-0.7 \mathrm{~V}$ and second reduction wave of the viologen unit which is initiated below $-0.5 \mathrm{~V}$. A small shoulder near $-0.1 \mathrm{~V}$ can be ascribed to the unexpected heterogeneitic part of the polymer layer that should be present to some extent when prepared by spin coating.

Cyclic voltammogram of the TEMPO-norbornene polymer showed a reversible wave near $1.26 \mathrm{~V}$, due to the $1 \mathrm{e}^{-}$oxidation of the TEMPO unit to the corresponding oxoammonium cation. ${ }^{15}$ The amount of charges consumed during the potential scan was $6 \mathrm{mC}$ based on the integration of the redox wave. The theoretical capacity for the polymer layer with the thickness of $210 \mathrm{~nm}$ was $26 \mathrm{mC}$. The calculated capacity was somewhat larger than the experimental capacity, suggesting the presence of a portion of the polymer layer that is incapable of undergoing the redox reaction, due probably to the partial lack of uniformity or the presence of defects. Nevertheless, the substantial redox capacity was sufficient to act as the reservoir of electrons that are transferred from the working electrode to effect the color change of the EC material with a capacity of $6 \mathrm{mC}$.

Using the PV-10-PSS complex/ITO/glass and the radical polymer/ITO/glass electrodes as the working and the counter electrodes respectively, the EC cell was fabricated as shown in Scheme 2. Incorporation of the radical polymer in the PV10PSS-based EC cell is intended (i) to lower the driving voltage and, consequently, the driving energy, in order to allow rapid switching based on the fast redox process of the radical centers and (ii) to show the color changes of the EC electrode alone by transmittance, featured by the transparency of the device. The transparent radical polymer allowed the simultaneous monitoring of the color changes by spectroscopy and electrochemical properties of the EC cell. The color of the EC cell changed, accompanied by the electrochemical reaction of PV10-PSS on the working electrode. The color change was recorded using the absorbance at $550 \mathrm{~nm}$ (Figure 2). The driving voltage of the EC cell is supposed to be $-1.4 \mathrm{~V}$, based on the gap between the redox potentials of PV10-PSS and the radical polymer.

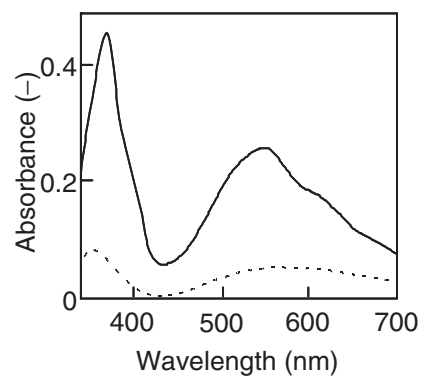

Figure 2. Spectral features of the EC cell in Scheme 2 obtained for the fully oxidized (dotted curve) and reduced (solid curve) states.
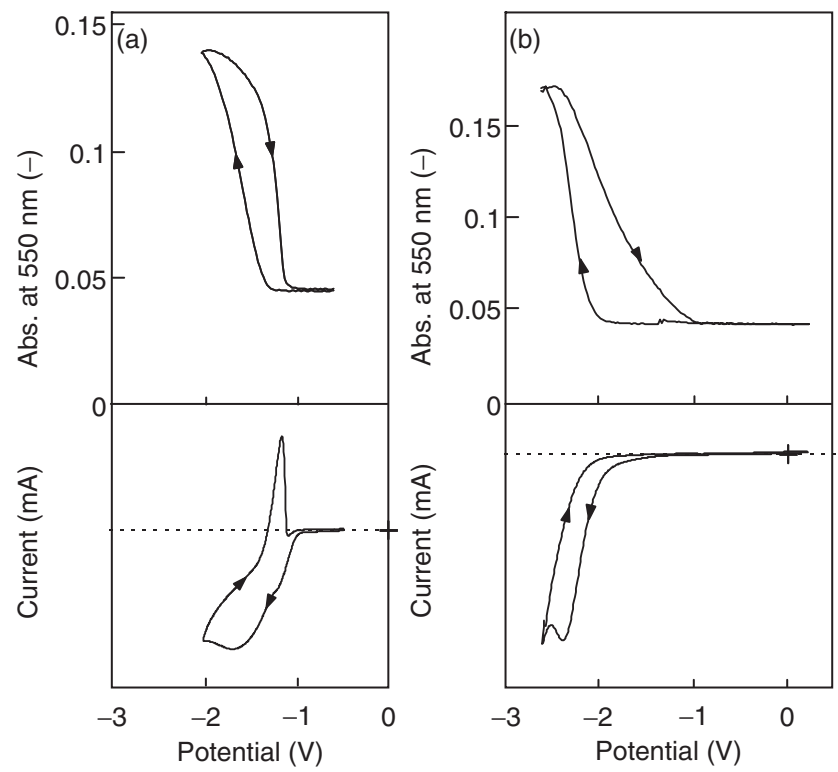

Figure 3. Spectroelectrochemical properties of the EC cell in Scheme 2 in the presence (a) and the absence (b) of the radical polymer on the counter electrode. The potential of the PV10-PSS/ITO/glass electrode was set versus the counter electrode, and scanned at a rate of $10 \mathrm{mV} / \mathrm{s}$.

Figure 3(a) shows the EC behavior of the device, which demonstrates that the color change is initiated by applying a voltage near $-1.4 \mathrm{~V}$. The amount of charges consumed during the color changes was almost equal to the amount of the redox sites in the PV10-PSS layer, which suggested the highly efficient electron injection to and rejection from the complex electrode. Note that an excess amount of the radical polymer was loaded on the counter electrode, considering the lower redox efficiency of the radical polymer side (vide supra). These results also supported that the redox reactions on working and counter electrodes occurred in the device to work as a doubleredox type EC cell. The larger reductive charge than the oxidative charge in Figure 3(a) is ascribed to the presence of residual $\mathrm{O}_{2}$ in the cell, based on the preliminary experiment that exhaustive deaeration leads to a more symmetric voltammogram. The effect of contaminated $\mathrm{O}_{2}$ on the cell performance is, however, beyond the scope of this research and will be reported in the future. In a control experiment, the EC cell without the electroactive material on the counter electrode showed a widely spread voltammogram in terms of applied voltages and hence the color change required a larger overpotential (Figure 3(b)), indicating the high resistance of the cell and the presence of undefined reactions at the counter electrode.

As for battery applications, one of the important factors to improve the property is to increase the energy density, and for this purpose, electroactive materials for cathodes and anodes are chosen with a large gap between their redox potentials. The design concept of the EC device is, on the other hand, to lower the driving voltage $\Delta V$ to cause the color changes. The work necessary to switch from one redox states to another is given by $\int \Delta V i(t) \mathrm{d} t$, where $\Delta V$ is the voltage applied to switch the 

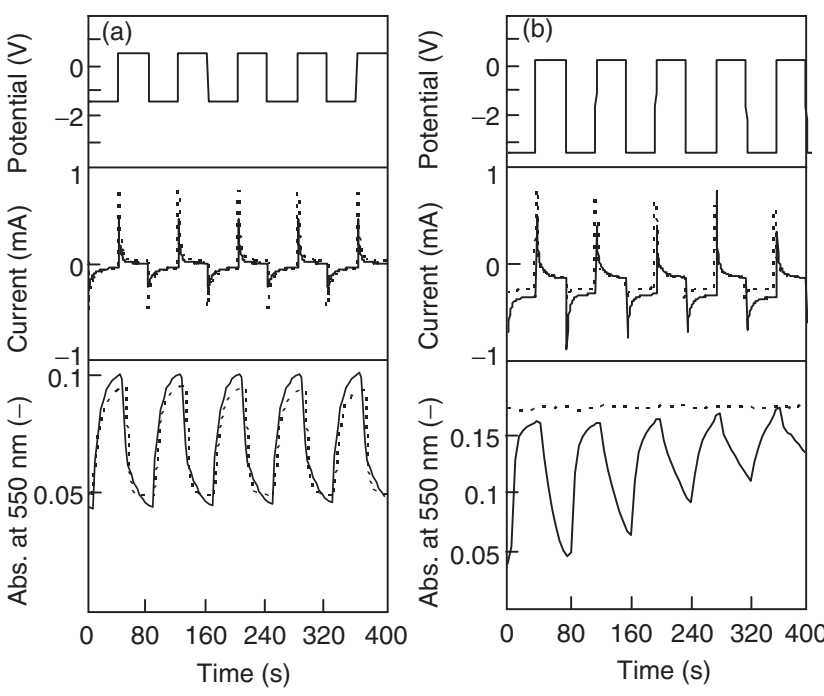

Figure 4. Spectroelectrochemical response of the EC cell in Scheme 2 in the presence (a) and the absence (b) of the radical polymer on the counter electrode. Potential pulses (top) were applied to the PV10PSS/ITO electrode versus the counter electrode, and the resulting current (middle) and absorbance at $550 \mathrm{~nm}$ (bottom) were recorded. The solid curves in the bottom figure represent the response obtained for the first 5 circles, and the dotted surves correspond to the response obtained after 50 cycles.

device and $i(t)$ is the time-dependent current. Figure 4 shows the relationship between the current and absorbance changes when a constant voltage was applied to switch the device. In the presence of the radical polymer at the counter electrode, the reversible and complete coloration and decoloration cycle was effected by applying a voltage of $-1.4 \mathrm{~V}$ and $0.5 \mathrm{~V}$, respectively. Like the prussian blue-based EC cell reported before, ${ }^{12}$ the driving voltage significantly decreased by using the radical polymer as the counter electrode-active material. The decoloration was readily initiated by applying short circuit conditions, i.e., at a driving voltage of $0 \mathrm{~V}$, although it proceeded slowly to take $c a .1 \mathrm{~min}$. The charged state was maintained at open circuit conditions. In the absence of the radical polymer on the counter electrode, the color change did not occur at such a low voltage; by applying a much larger voltage, however, the cell worked slowly and unstably (Figure 4(b)). The driving voltage was $-2.5 \mathrm{~V}$ and $1 \mathrm{~V}$ for the coloration/decoloration cycles. Necessity to apply high overpotentials also indicates the high resistance of the cell, which leads to the degradation after repeated cycles. These results suggested that lowering the driving voltages effectively improved the cycle performance, allowing even for the all organic cell to work for sufficiently long time.

Figure 5 shows the relationship between the chronopotentiometric response and the absorbance changes of the EC cell at $550 \mathrm{~nm}$. Electrolytic reduction of PV10-PSS was accompanied by the increase in the absorbance at $550 \mathrm{~nm}$ which changed almost linearly as a result of the constant current electrolysis. The charging/discharging curve exhibited a plateau voltage near $-1.4 \mathrm{~V}$ vs. the radical polymer/ITO as the counter electrode, which agreed well with the difference between the

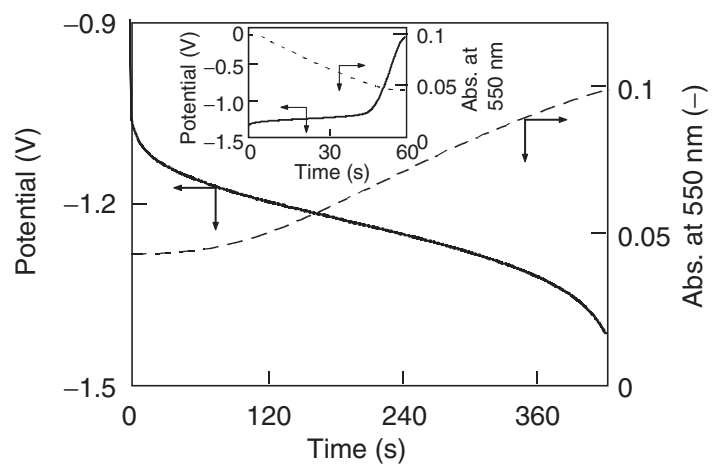

Figure 5. The absorbance change at $550 \mathrm{~nm}$ (dotted curve) accompanied by the constant-current chronopotentiometry (solid curve) at a current density of $1.1 \times 10^{-2} \mathrm{~mA} / \mathrm{cm}^{2}$ for the charging (coloration) process. Inset: the absorbance change at $550 \mathrm{~nm}$ at $2.2 \times 10^{-3} \mathrm{~mA} /$ $\mathrm{cm}^{2}$ for the discharging (decoloration) process.

(a)

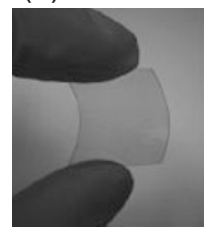

Figure 6. Photographs of the PV10-PSS film (a) and PV10-PSS based EC cell (b) on the ITO/PET electrodes.

potentials of the redox active materials on the working and the counter electrodes. By changing the polarity of the current, the absorbance at $550 \mathrm{~nm}$ that corresponds to the red-purple color of PV10-PSS decreased again, demonstrating the oxidation into the original PV10-PSS state. These results clearly supported the battery-like property of the EC cell.

Another curious feature is the capability of fabricating a flexible device using the ITO/PET electrode as the substrate of the polymer layers. By simply coating PV10-PSS and the radical polymers on the ITO/PET electrode, similar electrochemical behaviors and EC properties have preliminarily been obtained with the flexible EC cell (Figure 6), which demonstrated the advantage of using organic polymers on both electrodes. Further attempts to lower the inner resistance of the flexible device are underway, with a view to improve the switching rate performance.

In conclusion, we fabricated the all organic EC cell using the PV10-PSS complex as the EC material and the TEMPOradical polymer as the counter electroactive material, and confirmed the battery-like behavior associated with the coloration/decoloration process. The radical polymer at the counter electrode allowed the device to work at remarkably low driving voltages.

Acknowledgment. This work was partially supported by Grants-in-Aid for Scientific Research (Nos. 19105003 and 19655043) and the Global COE Program from MEXT, Japan. This work was carried out at the Advanced Research Institute for Science and Engineering, Waseda University. 
Received: March 24, 2008 Accepted: May 9, 2008 Published: July 2, 2008

\section{REFERENCES}

1. K. Bange and T. Gambke, Adv. Mater., 2, 10 (1990).

2. R. J. Mortimer, Chem. Soc. Rev., 26, 147 (1997).

3. M. Orellana, P. Arriola, R. D. Rio, R. Schrebler, R. Cordova, F. Scholz, and H. Kahlert, J. Phys. Chem. B, 109, 15483 (2005).

4. P. Varshney, M. Deepa, S. A. Agnihotry, and K. C. Ho, Sol. Energy Mater. Sol. Cells, 79, 449 (2003).

5. N. Kobayashi, S. Miura, and M. Nishimura, Sol. Energy Mater. Sol. Cells, 92, 136 (2008).

6. H. Urano, S. Sunohara, H. Ohtomoc, and N. Kobayashi, J. Mater. Chem., 14, 2366 (2004).

7. J. Stepp and J. B. Schlenoff, J. Electrochem. Soc., 144, L155 (1997).
8. G. Zotti, S. Zecchin, B. Vercelli, A. Berlin, S. Grimoldi, R. Bertoncello, and L. Milanese, J. Electroanal. Chem., 580, 330 (2005).

9. H. Ohno, N. Hosoda, and E. Tsuchida, Makromol. Chem., 184, 1061 (1983).

10. J. Zhang, T. Abe, and M. Kaneko, J. Electroanal. Chem., 438, 133 (1997).

11. E. Tsuchida and K. Abe, Adv. Polym. Sci., 45, 1 (1982).

12. Y. Takahashi, K. Oyaizu, K. Honda, and H. Nishide, J. Photopolym. Sci. Technol., 20, 29 (2007).

13. Y. Yonekuta, K. Oyaizu, and H. Nishide, Chem. Lett., 36, 866 (2007).

14. Y. Yonekuta, K. Honda, and H. Nishide, J. Am. Chem. Soc., 129, 14128 (2007).

15. T. Suga, H. Konishi, and H. Nishide, Chem. Commun., 1730 (2007).

16. H. Nishide and T. Suga, Electrochem. Soc. Interface, 14, 32 (2005).

17. T. Suga, Y.-J. Pu, K. Oyaizu, and H. Nishide, Bull. Chem. Soc. Jpn., 77, 2203 (2004).

18. H. Nishide and K. Oyaizu, Science, 319, 737 (2008).

19. C. Tanyeli and A. Gusmus, Tetrahedron Lett., 44, 1639 (2003). 